\title{
SOME CONVEXITY PROPERTIES OF THE DISTRIBUTION OF LOWER $k$-RECORD VALUES WITH EXTENSIONS
}

\author{
Mahdi Alimohammadi \\ Department of Statistics, \\ University of Isfahan, \\ Isfahan, 81746-73441, \\ Iran \\ Mohammad Hossein Alamatsaz \\ Department of Statistics, \\ University of Isfahan, \\ Isfahan, 81746-73441, \\ Iran \\ ERHARD CRAMER \\ Institute of Statistics, \\ RWTH Aachen University, \\ D-52056 Aachen, \\ Germany \\ Email: erhard.cramer@rwth-aachen.de
}

\begin{abstract}
Unimodality and strong unimodality of the distribution of ascendingly ordered random variables have been extensively studied in the literature, whereas these properties have not received much attention in the case of descendingly ordered random variates. In this paper, we show that log concavity of the reversed hazard rate implies that of the density function. Using this fundamental result, we establish some convexity properties of such random variables. To do this, we first provide a counterexample showing that a claim of Basak \& Basak [7] about the lower record values is not valid. Then, we provide conditions under which unimodality properties of the distribution of lower $k$-record values would hold. Finally, some extensions to dual generalized order statistics in both univariate and multivariate cases are discussed.
\end{abstract}

\section{INTRODUCTION}

Convex and concave functions play an important role in statistics, probability, and especially in reliability theory. Several aspects such as unimodality, strong unimodality, and logconcavity have been extensively discussed. A cumulative distribution function (cdf) $F$ is said to be unimodal if $F$ is either convex, concave, or a value $a \in \mathbb{R}$ exists such that $F$ is convex on 
$(-\infty, a)$ and concave on $(a, \infty)$. Assuming that $F$ has a probability density function (p.d.f.) $f$, this means that $f$ is either (i) non-decreasing, (ii) non-increasing, or (iii) non-decreasing on $(-\infty, a)$ and non-increasing on $(a, \infty)$, respectively. Note that these three properties may serve as a definition of unimodality for non-negative functions $h$. Alternatively, one may define unimodality of a non-negative function $h$ via convexity of the sets $\{x: h(x) \geq \alpha\}$ for any $\alpha>0$ (cf. An [4]). A cdf $F$ is said to be strongly unimodal if its convolution with any unimodal distribution is unimodal. Thus, any strongly unimodal distribution is unimodal but the converse is not necessarily true. The famous result of Ibragimov [20] linked this property to logconcavity of the corresponding p.d.f. $f$. He showed that a non-degenerate cdf $F$ is strongly unimodal if, and only if, it has a logconcave p.d.f. $f$. A non-negative function $h: \mathbb{R}^{n} \longmapsto \mathbb{R}_{+}$is said to be logconcave if $h(\alpha \mathbf{x}+(1-\alpha) \mathbf{y}) \geq[h(\mathbf{x})]^{\alpha}[h(\mathbf{y})]^{1-\alpha}$, for all $\mathbf{x}, \mathbf{y} \in \mathbb{R}^{n}$ and $\alpha \in(0,1)$. At this point, it has to be mentioned that logconcavity of the density function $f$ is equivalent to the property that $f$ is a Pólya frequency function of order $2\left(\mathrm{PF}_{2}\right)$ (see Karlin [24]). Barlow \& Proschan [6, p. 76] proved that the $P F_{2}$ property of $f$ leads to increasing failure rate (IFR) property of its distribution. An extensive survey on these concepts is provided by the monograph of Dharmadhikari \& Joag-Dev [17].

Unimodality and strong unimodality of the distribution of ascendingly ordered random variables have been extensively studied (see, e.g., Alam [1], Huang \& Ghosh [19], Raqab \& Amin [29], Basak \& Basak [7], Aliev [2], Cramer, Kamps \& Rychlik [15], and Cramer [13]). Since generalized order statistics (GOSs), defined by Kamps ( $[21,22])$, contain all the models discussed by the above authors, recent results of Chen, Xie \& Hu [12] and Alimohammadi \& Alamatsaz [3] concerning GOSs have unified most of the previous findings. On the other hand, the case of descendingly ordered random variables has not received much attention so far.

In another direction, Burkschat, Cramer \& Kamps [9] introduced a dual model that enables a common approach to descendingly ordered random variables called dual generalized order statistics (DGOSs) including reversed ordered order statistics and lower $k$-record values. Let $F$ be an absolutely continuous cdf with p.d.f. $f$ and define $\bar{F}=1-F$. Then, random variables $X_{(r, n, \tilde{m}, k)}^{d}, r=1,2, \ldots, n$, are called DGOSs based on $F$ if their joint density function is given by

$$
f_{X_{(1, n, \tilde{m}, k)}^{d}, \ldots, X_{(n, n, \tilde{m}, k)}^{d}}\left(x_{1}, \ldots, x_{n}\right)=k\left(\prod_{j=1}^{n-1} \gamma_{j}\right)\left(\prod_{i=1}^{n-1}\left[F\left(x_{i}\right)\right]^{m_{i}} f\left(x_{i}\right)\right)\left[F\left(x_{n}\right)\right]^{k-1} f\left(x_{n}\right),
$$

for all $F^{-1}(1-) \geq x_{1} \geq x_{2} \geq \cdots \geq x_{n}>F^{-1}(0)$, where $n \in \mathbb{N}, k>0$ and $m_{1}, \ldots, m_{n-1} \in$ $\mathbb{R}$ are such that $\gamma_{r}=k+n-r+\sum_{j=r}^{n-1} m_{j}>0$ for all $r \in\{1, \ldots, n-1\}$, and $\tilde{m}=$ $\left(m_{1}, \ldots, m_{n-1}\right)$, for $n \geq 2(\tilde{m} \in \mathbb{R}$ is arbitrary for $n=1)$.

There exist some representations for the marginal density functions of GOSs (see, e.g., Kamps [21,22], Kamps \& Cramer [23], Cramer \& Kamps [14], Cramer, Kamps \& Rychlik [15]). Cramer, Kamps \& Rychlik [15] established a representation of the p.d.f. of the GOS $X_{(r, n, \tilde{m}, k)}$ in the form

$$
f_{X_{(r, n, \tilde{m}, k)}}(x)=c_{r-1}[\bar{F}(x)]^{\gamma_{r}-1} g_{r}(F(x)) f(x), \quad x \in \mathbb{R},
$$

where $c_{r-1}=\prod_{i=1}^{r} \gamma_{i}, r=1, \ldots, n, \gamma_{n}=k$, and $g_{r}$ is a particular Meijer's $G$-function (see Eq. (2.6) in Cramer, Kamps \& Rychlik [15]). Tavangar \& Asadi [31] rediscovered this representation by presenting an integral representation of $g_{r}$. Analogously, Burkschat, Cramer \& Kamps [9] gave a representation for the p.d.f. of DGOSs. In particular, they stated that if 
$X_{(r, n, \tilde{m}, k)}, r=1, \ldots, n$, are GOSs based on an absolutely continuous cdf $G$ and $X_{(r, n, \tilde{m}, k)}^{d}$, $r=1, \ldots, n$, are DGOSs based on an absolutely continuous cdf $F$, then

$$
\left(F\left(X_{(r, n, \tilde{m}, k)}^{d}\right)\right)_{1 \leq r \leq n} \stackrel{d}{=}\left(1-G\left(X_{(r, n, \tilde{m}, k)}\right)\right)_{1 \leq r \leq n} .
$$

So, from (2) and (3) we arrive at the marginal p.d.f. of the DGOS $X_{(r, n, \tilde{m}, k)}^{d}$ as:

$$
f_{X_{(r, n, \tilde{m}, k)}^{d}}(x)=c_{r-1}[F(x)]^{\gamma_{r}-1} g_{r}(\bar{F}(x)) f(x), \quad x \in \mathbb{R},
$$

where $c_{r-1}$ and $g_{r}$ are as in (2). For $m_{1}=m_{2}=\cdots=m_{n-1}=m$, the p.d.f. (4) simplifies to

$$
f_{X_{(r, n, m, k)}^{d}}(x)=\frac{c_{r-1}}{(r-1) !}[F(x)]^{\gamma_{r}-1} h_{m}^{r-1}(F(x)) f(x), \quad x \in \mathbb{R},
$$

where

$$
h_{m}(x)=\left\{\begin{array}{ll}
\frac{1}{m+1}\left(1-x^{m+1}\right), & m \neq-1 \\
-\ln (x), & m=-1
\end{array}, \quad x \in[0,1),\right.
$$

(see also Kamps $[21,22]$ ).

Notice that, for $m_{1}=\cdots=m_{n-1}=-1$ and $k \in \mathbb{N}$, the models of GOSs and DGOSs equal the upper and lower $k$-record models in distribution, respectively. Thus, the p.d.f. of the lower $k$-record value $X_{(r, n,-1, k)}^{d}$ is given by

$$
f_{X_{(r, n,-1, k)}^{d}}(x)=\frac{k^{r}}{(r-1) !}[F(x)]^{k-1}[-\ln F(x)]^{r-1} f(x), \quad x \in \mathbb{R},
$$

(see, e.g., Arnold, Balakrishnan \& Nagaraja [5] and Nevzorov [26]).

In this paper, we show that logconcavity of the reversed hazard rate $r=f / F$ implies that of the corresponding density function. Using this fundamental result, we establish some convexity properties of such random variables. To do this, we first provide a counterexample showing that a claim of Basak \& Basak [7, Remark, p. 396] about the lower record values is not valid. Then, we provide conditions under which unimodality properties of the distribution of lower $k$-record values hold. In this regard, the notion of local unimodality of the distributions for ordered random variables is dealt with in this paper for the first time. Finally, some extensions to DGOSs in both univariate and multivariate cases are discussed.

\section{CONVEXITY PROPERTIES OF LOWER $k$-RECORD VALUES BASED ON THE REVERSED HAZARD RATE}

In view of the growing importance of reversed hazard rate in reliability analysis and stochastic modeling, a wide interest has been shown in investigating its properties (see, e.g., Block, Savits \& Singh [8], Finkelstein [18], Marshall \& Olkin [25] and Shaked \& Shanthikumar [30]). In what follows, we first provide two fundamental interesting results for reversed hazard rates. Here, $L=\inf \{x: F(x)>0\}$ and $U=\sup \{x: F(x)<1\}$ denote the lower and upper bounds of the support of the underlying cdf $F$, respectively. Hence, the interval of support is given by $(L, U)$ where $-\infty \leq L<U \leq \infty$.

Block, Savits \& Singh [8] have shown that the support must be $(-\infty, U)$ with $U<\infty$ provided that the reversed hazard rate is a non-decreasing function on $(L, U)$. Lemma 2.1 provides an extension of this result to unimodal reversed hazard rates when $L$ is supposed to be finite. 
Lemma 2.1: Let $L$ be finite. If the reversed hazard rate $r=f / F$ is unimodal, then it has to be non-increasing.

PROOF: As pointed out in the introduction, a unimodal reversed hazard rate $r$ can result in three cases: (i) $r$ is non-decreasing; (ii) $r$ is first non-decreasing and then non-increasing and, finally; and (iii) $r$ is non-increasing. In cases (i) and (ii), $r$ must be non-decreasing on an interval $(L, \alpha)$ for some $\alpha \in(L, U)$ with $F(\alpha)>0$. Then, the reversed hazard rate of the right truncated cdf $F_{\alpha}$ defined by $F_{\alpha}=F / F(\alpha)$ with support $(L, \alpha)$ has the same reversed hazard rate as $F$ on $(L, \alpha)$. Since $r$ is assumed to be non-decreasing on $(L, \alpha)$, Theorem 2.1 of Block. Savits \& Singh [8] proves that $L=-\infty$ must hold which contradicts our assumption. Therefore, cases (i) and (ii) can not occur for a unimodal $r$. Thus, the desired result follows.

It is worth mentioning that Pellerey, Shaked \& Zinn [27] proved an analogous result of Lemma 2.1 for hazard rates under the stronger condition of logconcavity of hazard rates for non-negative random variables. The corresponding proof is quite different and lengthy. Here, we have relaxed the logconcavity condition to unimodality.

Chandra \& Roy [10] showed that the reversed hazard rate is non-increasing when its p.d.f. $f$ is logconcave. In the following lemma, we consider a reversed implicative relationship between reversed hazard rate and density function.

Lemma 2.2: Suppose that the reversed hazard rate $r$ is logconcave. If either

(i) $L$ is finite

(ii) or $L=-\infty$ and $r$ is non-increasing,

then the p.d.f. $f$ is logconcave.

Proof: From Lemma 2.1, we have that $r$ is non-increasing for finite $L$. Therefore, we can assume that $r$ is both logconcave and non-increasing whatever so is $L$. Then, we rewrite the reversed hazard rate formula as $f=r \cdot F$, so that, for $x \in(L, U)$,

$$
\ln f(x)=\ln r(x)+\ln F(x)=\ln r(x)-R(x),
$$

where $R(x)=\int_{x}^{+\infty} r(y) d y$ is called the reversed hazard function. Since $r$ is non-increasing, $R$ must be convex on $(L, U)$ which means that $-R$ is concave. Since the sum of two concave functions is concave, (7) yields concavity of $\ln f$ and, hence, we have the desired result.

It should be mentioned that logconcavity of the reversed hazard rate is not a necessary condition for logconcavity of the p.d.f. As an example, one may consider the Rayleigh distribution with cdf $F(x)=1-\exp \left(-x^{2} / 2\right), x>0$. Obviously, the p.d.f. is logconcave. However, the reversed hazard rate $r$ with $r(x)=\frac{x}{\exp \left(x^{2} / 2\right)-1}, x>0$, is not logconcave.

Although the restriction of $L$ being finite is required in Lemma 2.1, Lemmas 2.1 and 2.2 can be applied to many distributions such as lifetime distributions with finite left endpoint of support. Furthermore, several distributions with $L=-\infty$ such as normal, logistic, and Gumbel as well as distributions with finite $L$ such as gamma and Weibull have decreasing reversed hazard rate. For other examples and relations, we refer the reader to Chechile [11].

Now, we focus on unimodality properties of the distribution of lower $k$-record values. Aliev [2] provided a counterexample showing that convexity of $1 / f$, as formulated by Basak $\&$ Basak [7], is not sufficient to conclude that the upper record values are unimodal. Now, we provide the following counterexample for the lower record values with a stronger condition 


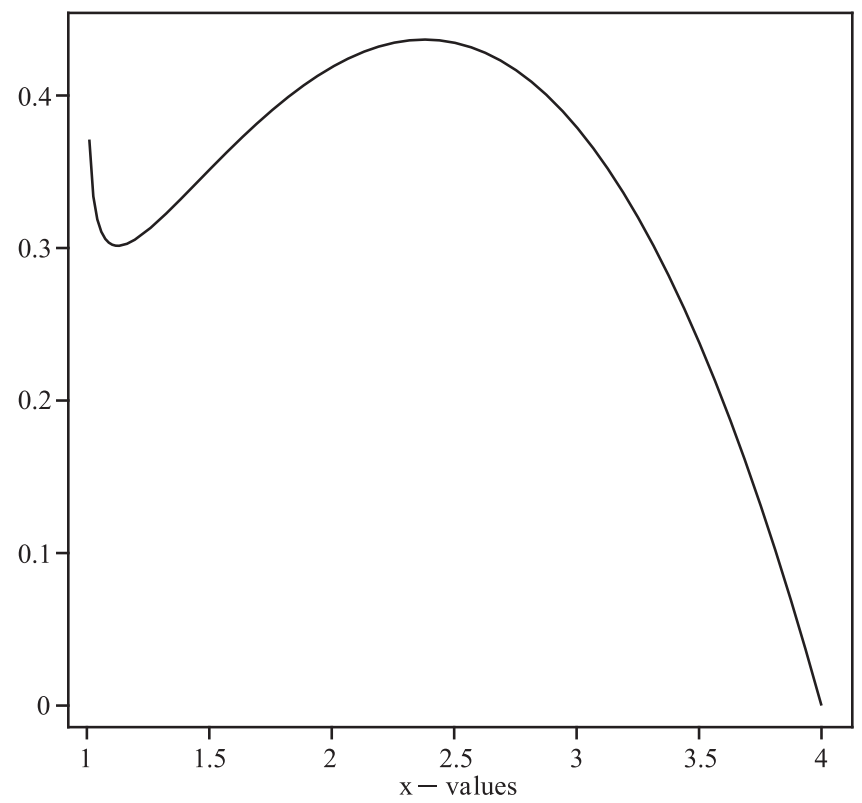

Figure 1. The p.d.f. of the second lower record value in Counterexample 2.3.

on $f$. Furthermore, this counterexample shows that strong unimodality of $F$ is not sufficient for strong unimodality of lower record values.

Counterexample 2.3: Suppose that in (6) $r=2, k=1$ and $f(x)=x^{2} / 21,1 \leq x \leq 4$. Clearly, $f$ is logconcave and so $1 / f$ is convex. However, Figure 1 shows that the second lower record value is not unimodal and therefore, it is not strongly unimodal.

Unfortunately, the distribution of $k$-record values can hardly be unimodal. The situation is even worse for lower records than for the upper ones. For upper $k$-record values, Alimohammadi \& Alamatsaz [3] established the result assuming additionally the monotonicity of $f$. But, this assumption does not work for the lower $k$-record values (for details we refer to Remark 2.5). In such a situation, however, we shall show below that for local unimodality of the lower and upper $k$-record values, the condition of $1 / f$ being convex is sufficient. Furthermore, the corresponding interval for local unimodality is specified.

TheOREM 2.4: Let the p.d.f. $f$ of the cdf $F$ be such that $1 / f$ is convex on $(L, U)$. Then, all lower (upper) $k$-record values have locally unimodal distributions on $\left(F^{-1}\left(e^{-1}\right), U\right)$ $\left(\left(L, F^{-1}\left(1-e^{-1}\right)\right)\right)$.

Proof: Let $f_{X_{(r, n,-1, k)}^{d}}^{\prime}$ denote the right derivative of $f_{X_{(r, n,-1, k)}^{d}}$. So, from (6) we have

$$
f_{X_{(r, n,-1, k)}^{d}}^{\prime}(x)=G_{1}(x)\left[\frac{k-1}{F(x)}+\frac{(r-1)}{F(x) \ln F(x)}+\frac{f^{\prime}(x)}{f^{2}(x)}\right]=G_{1}(x) \cdot w(x), \quad \text { say, }
$$

where $G_{1}(x)=\frac{k^{n}}{(r-1) !}[F(x)]^{k-1}[-\ln F(x)]^{r-1} f^{2}(x)$. Note that convexity of $1 / f$ implies that $f$ is right differentiable and $f^{\prime} / f^{2}$ is non-increasing. Now, $w(x)$ is non-increasing in $x$ 
provided that $F(x) \ln F(x)$ is non-decreasing in $x$, i.e., when

$$
(F(x) \ln F(x))^{\prime}=f(x) \ln F(x)+f(x) \geq 0 .
$$

Thus, $\ln F(x) \geq-1$ and hence $x \geq F^{-1}\left(e^{-1}\right)$. Now $G_{1}(x) \geq 0$ and $w(x)$ is non-increasing on $\left(F^{-1}\left(e^{-1}\right), U\right)$. Thus $f_{X_{(r, n,-1, k)}^{d}}^{\prime}$ changes sign at most once as $x$ moves from $L$ to $U$ and any sign change has to be from positive to negative. Clearly, this implies that $f_{X_{(r, n,-1, k)}^{d}}$ is unimodal.

For upper records, the result follows immediately from the p.d.f. of the upper $k$-record values and its derivative (9) as given below:

$$
f_{X_{(r, n,-1, k)}}(x)=\frac{k^{n}}{(r-1) !}[\bar{F}(x)]^{k-1}[-\ln \bar{F}(x)]^{r-1} f(x), \quad x \in \mathbb{R}
$$

and

$$
f_{X_{(r, n,-1, k)}}^{\prime}(x)=G_{2}(x)\left[\frac{-(k-1)}{\bar{F}(x)}+\frac{(r-1)}{\bar{F}(x)(-\ln \bar{F}(x))}+\frac{f^{\prime}(x)}{f^{2}(x)}\right]
$$

where $G_{2}(x)=\frac{k^{n}}{(r-1) !}[\bar{F}(x)]^{k-1}[-\ln \bar{F}(x)]^{r-1} f^{2}(x)$.

Remark 2.5: We can rewrite (8) and (9) as:

$$
f_{X_{(r, n,-1, k)}^{d}}^{\prime}(x)=\frac{G_{1}(x)}{F(x)}\left[(k-1)+\frac{(r-1)}{\ln F(x)}+\frac{f^{\prime}(x)}{f^{2}(x)} F(x)\right]
$$

and

$$
f_{X_{(r, n,-1, k)}}^{\prime}(x)=\frac{G_{2}(x)}{\bar{F}(x)}\left[-(k-1)+\frac{(r-1)}{(-\ln \bar{F}(x))}+\frac{f^{\prime}(x)}{f^{2}(x)} \bar{F}(x)\right] .
$$

Comparing (10) with (11), we find that even by adding the condition of monotonicity of $f$, the quantity inside the brackets on the right-hand side of (10) cannot be non-increasing in $x$, but this is the case for (11).

Counterexample 2.3 shows that lower $k$-records need not be unimodal whereas Theorem 2.4 illustrates that the property of unimodality holds at least on the right tail of the distribution. Theorem 2.6 proves that unimodality holds for lower and upper $k$-record values from a uniform distribution (see also Remark 3.3).

THEOREM 2.6: The distribution of each lower and upper $k$-record value from a uniform distribution is unimodal.

Proof: The assertion follows from (10) and (11) using the same argument as in the proof of Theorem 2.4, because $f^{\prime}(x)=0, x \in(L, U)$, and, thus, the third troublesome term inside the brackets of (10) and (11) cancels out.

Furthermore, it should be mentioned that the p.d.f. of lower and upper record values from a uniform distribution are not logconcave in general though they are unimodal (see also Remark 3.3). This is illustrated by the following counterexample. 
Counterexample 2.7: Consider the second lower record value from a uniform distribution. Its p.d.f. is given by (see (6))

$$
f_{X_{(2, n,-1,1)}^{d}}(x)=-\ln (x), \quad x \in(0,1] .
$$

The second derivative of the logarithm of the p.d.f. is given by $\left(\ln f_{X_{(2, n,-1,1)}^{d}}\right)^{\prime \prime}(x)=$ $-\frac{\ln (x)+1}{x^{2}(\ln (x))^{2}}, x \in(0,1]$, which obviously has a sign change at $x=e^{-1}$ from positive to negative. Thus, $\ln f_{X_{(2, n,-1,1)}^{d}}$ is convex on $\left(0, e^{-1}\right)$ and concave on $\left(e^{-1}, 1\right)$. Hence, $f_{X_{(2, n,-1,1)}^{d}}$ is not logconcave. Similar arguments apply to upper records.

To obtain the next results of this section, we need the following lemma.

Lemma 2.8 (An [4]): Let $h: \mathbb{R} \longmapsto \mathbb{R}_{+}$be a measurable function and $\{x: h(x)>0\}=$ $(a, b)$. If $h$ is logconcave on $(a, b)$, then both $H_{1}(x)=\int_{a}^{x} h(y) d y$ and $H_{2}(x)=\int_{x}^{b} h(y) d y$ are also logconcave on $(a, b)$.

The next theorems deal with logconcavity of distributions of lower $k$-record values.

THEOREM 2.9: Let the reversed hazard rate of $F$ be logconcave. If either

(i) L is finite

(ii) or $L=-\infty$ and $r$ is non-increasing,

then the joint p.d.f. of lower $k$-record values is logconcave.

Proof: Let $m_{1}=\cdots=m_{n-1}=-1$ in (1). Then, according to Lemmas 2.2 and 2.8 , we have

logconcavity of $r \Rightarrow$ logconcavity of $f \Rightarrow$ logconcavity of $F$.

So, the joint density in (1) becomes a product of univariate logconcave functions. Since logconcavity is preserved under products, the assertion holds. values.

The preceding lemma is now applied to the marginal distributions of lower $k$-record

Lemma 2.10 (Prékopa [28]): Suppose that $h: \mathbb{R}^{n} \times \mathbb{R}^{m} \longmapsto \mathbb{R}_{+}$is a logconcave function and $\eta(\boldsymbol{x})=\int_{\mathbb{R}^{m}} h(\boldsymbol{x}, \boldsymbol{y}) d \boldsymbol{y}$ is finite for each $\boldsymbol{x} \in \mathbb{R}^{n}$. Then, $\eta$ is logconcave on $\mathbb{R}^{n}$.

THEOREM 2.11: Any marginal p.d.f. of lower $k$-record values is logconcave and so strongly unimodal if either of the following satisfies:

(i) $L$ is finite and the reversed hazard rate of $F$ is logconcave, or $L=-\infty$ and the reversed hazard rate of $F$ is logconcave and non-increasing.

(ii) The p.d.f. $f$ and the reversed hazard function $R$ are both logconcave.

Proof: (i) The assertion follows directly from Lemma 2.10 and Theorem 2.9.

(ii) Since $R=-\ln F$ and logconcavity of $f$ implies logconcavity of $F$ (Lemma 2.8), the result follows by the conditions of the theorem and the p.d.f. given in (6). 
Remark 2.12: Logconcavity of $r$ implies logconcavity of $f$ (Lemma 2.2) and $R$ (Lemma 2.8). So, the condition in (ii) is weaker than that of (i).

EXAMPLE 2.13: Clearly, the exponential distribution has a constant hazard rate, but its reversed hazard rate is decreasing. By straightforward calculations it can be seen that the exponential distribution does not satisfy the conditions of Theorem 2.11 part (i). In fact, its reversed hazard rate is logconvex. However, it satisfies part (ii) of Theorem 2.11. Consequently, lower $k$-record values from an exponential population are strongly unimodal and, thus, unimodal.

\section{EXTENSIONS TO DGOSS}

First, note that many results on DGOSs can be obtained from those of GOSs using the relationship presented in Eq. (3). But, in Theorem 3.4, we establish some stronger results than those resulting from the findings on logconcavity of GOSs. Theorems 3.1 and 3.6 are an adaption of results for GOSs established in Chen, Xie \& Hu [12] and Alimohammadi \& Alamatsaz [3], respectively. They are given for completeness and thus we omit their proofs.

TheOREm 3.1: Suppose that $X_{(r, n, \tilde{m}, k)}^{d}, r=1,2, \ldots, n$, are DGOSs based on an absolutely cdf $F$ with p.d.f. f. Then, the joint p.d.f. of DGOSs is logconcave if any of the following conditions is satisfied:

(i) $k \geq 1, m_{i} \geq 0, i=1, \ldots, n-1$ and $F$ is strongly unimodal.

(ii) $k>0, m_{i} \geq-1, i=1, \ldots, n-1$,

(a) $L$ is finite and the reversed hazard rate of $F$ is logconcave,

(b) $L=-\infty$ and the reversed hazard rate of $F$ is logconcave and non-increasing.

We need to recall the following lemma to obtain the main results of this section.

Lemma 3.2 (Alimohammadi \& Alamatsaz [3]): The functions $g_{r}, r=2, \ldots, n$, in (2) are logconcave for $m_{i} \geq 0, i=1, \ldots, n-1$.

Remark 3.3: Notice that the result of Lemma 3.2 can also be deduced using that $g_{r}=k_{r}^{-1} \cdot f_{r}$ where $f_{r}$ is the marginal density function of the $r$ th uniform GOS with parameters $m_{1}, \ldots, m_{r-1} \geq 0, k=1$, (or, equivalently, $\tilde{\gamma}_{1} \geq \cdots \geq \tilde{\gamma}_{r-1} \geq \tilde{\gamma}_{r}=1$ ) and $k_{r}=$ $\prod_{j=1}^{r} \tilde{\gamma}_{j}$ is an appropriate normalizing constant (here $\tilde{\gamma}_{j}=\gamma_{j}-\gamma_{r}+1,1 \leq j \leq r$ ). Hence, Corollary 4.1 of Cramer [13] yields logconcavity of $g_{r}$. Further, we can apply Lemma 4.5 in Cramer [13] in combination with the notion of multiplicative strong unimodality as introduced by Cuculescu \& Theodorescu [16]. This proves that the cdf of a uniform DGOS is always unimodal (cf. Theorem 2.6). Moreover, it is convex if the smallest gamma is included in $(0,1]$.

THEOREM 3.4: Any marginal p.d.f. of DGOSs is logconcave and so strongly unimodal if any of the following conditions is satisfied:

(i) Either conditions (i) or (ii) of Theorem 3.1 hold.

(ii) For $r=1$ and $m_{i} \in \mathbb{R}, i=1, \ldots, n-1$ :

(a) $\gamma_{r} \geq 1$ and $F$ is strongly unimodal,

(b) $\gamma_{r}>0, L$ is finite and the reversed hazard rate of $F$ is logconcave, 
(c) $\gamma_{r}>0, L=-\infty$ and the reversed hazard rate of $F$ is logconcave and nonincreasing.

(iii) For $r \geq 2$, the situations and conditions are the same as for $r=1$ but with $m_{i} \geq 0$.

Proof: $\quad$ (i) The assertion follows directly from Lemma 2.10 and Theorem 3.1.

(ii) The result in (a) follows by (4). Also, parts (b) and (c) hold by rewriting (4) in the following form with $r=1$ :

$$
f_{X_{(r, n, \tilde{m}, k)}^{d}}(x)=c_{r-1}[F(x)]^{\gamma_{r}} g_{r}(\bar{F}(x)) r(x), \quad x \in \mathbb{R} .
$$

(iii) Lemma 3.2 yields the result for $r \geq 2$.

Remark 3.5: The above results are stronger than those of Chen, Xie \& Hu [12]. They had only proved an analogous result to part (i) of our Theorem 3.4 for GOSs, while our findings contain two more results in parts (ii) and (iii) of the theorem for DGOSs. Their results were also incomplete for certain cases of the parameter values involved. This shortcoming is also clarified by our rather strong Counterexample 3.8 for DGOSs.

In the next theorem, we present some sufficient conditions to ensure unimodality of DGOSs.

TheOrem 3.6: Let $F$ be a cdf with p.d.f. f. Then, its DGOS is unimodal if any of the following conditions is satisfied:

(i) For $r=1$ and $m_{i} \in \mathbb{R}, i=1, \ldots, n-1$ :

(a) $0<\gamma_{r}<1$ and $f$ is right differentiable and non-increasing on its support,

(b) $\gamma_{r}=1$ and $f$ is unimodal,

(c) $1<\gamma_{r}$ and either $1 / f$ is convex or $f$ is right differentiable and non-decreasing on its support, and

(ii) For $r \geq 2$ :

(a) $0<\gamma_{r}<1, m_{i} \in \mathbb{R}, i=1, \ldots, n-1$, and $f$ is right differentiable and nonincreasing on its support,

(b) $\gamma_{r}=1, m_{1}=\cdots=m_{n-1}=-1$, and $f$ is right differentiable and non-increasing on its support,

(c) $1 \leq \gamma_{r}, 0 \leq m_{i}, i=1, \ldots, n-1$, and $1 / f$ is convex.

Remark 3.7: Alam [1] showed the unimodality of order statistics by convexity of $1 / f$. Then, Huang \& Ghosh [19] proved that order statistics from strongly unimodal distributions are strongly unimodal. According to Theorems 3.6 and 3.4, reversed ordered order statistics have also unimodal and strongly unimodal distributions when $1 / f$ is convex and $f$ is logconcave, respectively. Note that the parameters are given by $m_{1}=\cdots=m_{n-1}=0$ and $k=1$ but the p.d.f. for GOS and DGOS has the same structure. This reflects the symmetry in the case of order statistics, which is just a change in the population cdf from $F$ to $1-F(-\cdot)$.

The above theorems are stated by means of the notions of convexity and logconcavity of functions. In particular, the implications

(i) $\log$ concavity of $r \Rightarrow$ (ii) $\log c o n c a v i t y$ of $f \Rightarrow$ (iii)convexity of $1 / f \Rightarrow$ (iv) unimodality of $f$ are an important tool in the derivations. In order to illustrate these conditions, we present some examples. First, the logistic distribution satisfies (i) and, thus, (ii), (iii) and (iv). The 


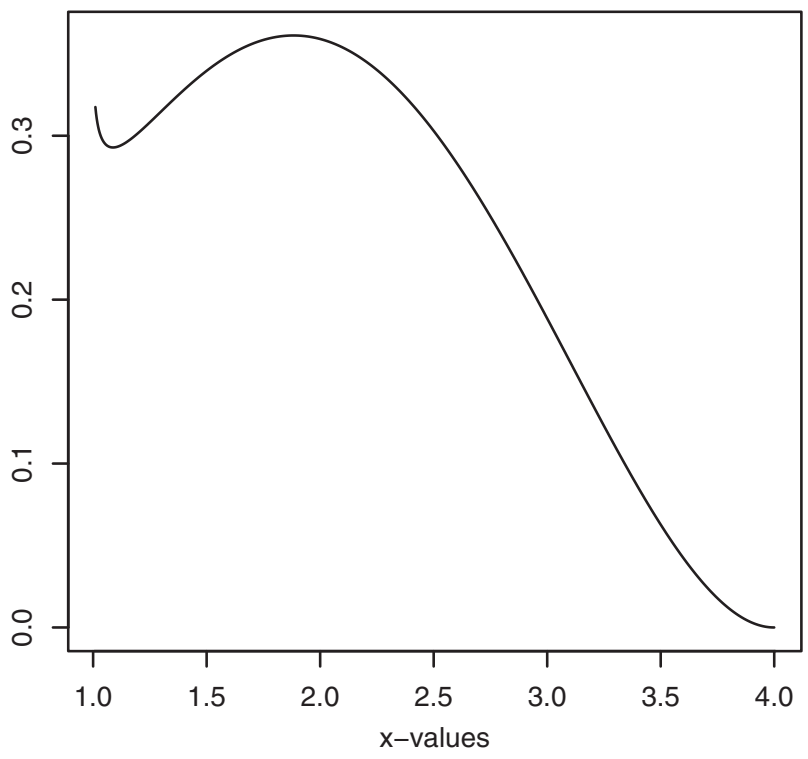

Figure 2. The p.d.f. of the third DGOS in Counterexample 3.8.

continuous uniform distribution satisfies (ii) but not (i). The Cauchy distribution satisfies (iii) and so (iv) but not (i) and (ii). Finally, the power distribution with parameter less than one only satisfies (iv).

We should note that Theorems 3.4 and 3.6 include most values of the parameters except for cases with $m_{i}<0$, for some $i \in\{1, \ldots, n-1\}$. However, the following counterexample presents a situation where Theorems 3.4 (iii) and 3.6 (ii) fail.

Counterexample 3.8: Let $n=4, \gamma_{4}=k=0.7, \quad m_{1}=m_{2}=m_{3}=-0.7$ and $f(x)=$ $x^{2} / 21,1 \leq x \leq 4$ in (5). Note that, $f$ satisfies not only the condition of $1 / f$ being convex, but also it is logconcave. However, Figure 2 shows that the third DGOS is not unimodal and therefore, it is not strongly unimodal.

It is worthwhile pointing out that this counterexample is stronger than that presented in Alimohammadi \& Alamatsaz [3], because the p.d.f. $f$ in their counterexample satisfies only the condition of $1 / f$ being convex.

\section{Acknowledgements}

The first author is grateful to the office of Graduate Studies of the University of Isfahan for their support.

\section{References}

1. Alam, K. (1972). Unimodality of the distribution of an order statistic. The Annals of Mathematical Statistics 43: 2041-2044.

2. Aliev, F.A. (2003). A comment on 'Unimodality of the distribution of record statistics'. Statistics and Probability Letters 64: 39-40.

3. Alimohammadi, M. \& Alamatsaz, M.H. (2011). Some new results on unimodality of generalized order statistics and their spacing. Statistics and Probability Letters 81: 1677-1682.

4. An, M.Y. (1998). Logconcavity versus logconvexity: a complete characterization. Journal of Economic Theory 80: 350-369.

5. Arnold, B.C., Balkrishnan, N. \& Nagaraja, H.N. (1998). Records. New York: Wiley. 
6. Barlow, R.E. \& Proschan, F. (1975). Statistical Theory of Reliability and Life Testing. New York: Holt, Rinehart and Winston.

7. Basak, P. \& Basak, I. (2002). Unimodality of the distribution of record statistics. Statistics and Probability Letters 56: 395-398.

8. Block, H., Savits, T.H. \& Singh, H. (1998). The reversed hazard rate function. Probability in the Engineering and Informational Sciences 12: 69-90.

9. Burkschat, M., Cramer, E. \& Kamps, U. (2003). Dual generalized order statistics. Metron 61: 13-26.

10. Chandra, N.K. \& Roy, D. (2001). Some results on reverse hazard rate. Probability in the Engineering and Informational Sciences 15: 95-102.

11. Chechile, R.A. (2011). Properties of reverse hazard functions. Journal of Mathematical Psychology 55: 203-222.

12. Chen, H., Xie, H. \& Hu, T. (2009). Log-concavity of generalized order statistics. Statistics and Probability Letters 79: 396-399.

13. Cramer, E. (2004). Logconcavity and unimodality of progressively censored order statistics. Statistics and Probability Letters 68: 83-90.

14. Cramer, E. \& Kamps, U. (2003). Marginal distributions of sequential and generalized order statistics. Metrika 58: 293-310.

15. Cramer, E., Kamps, U. \& Rychlik, T. (2004). Unimodality of uniform generalized order statistics, with applications to mean bounds. Annals of the Institute of Statistical Mathematics 56: 183-192.

16. Cuculescu, I. \& Theodorescu, R. (1998). Multiplicative strong unimodality. Australian and New Zealand Journal of Statistics 40: 205-214.

17. Dharmadhikari, S. \& Joag-Dev, K. (1988). Unimodality, Convexity, and Applications. Boston: Academic Press.

18. Finkelstein, M.S. (2002). On the reversed hazard rate. Reliability Engineering and System Safety 78: $71-75$.

19. Huang, J.S. \& Ghosh, M. (1982). A note on strong unimodality of order statistics. Journal of the American Statistical Association 77: 929-930.

20. Ibragimov, I.A. (1956). On the composition of unimodal distributions. Theory of Probability and Its Applications 1: 255-260.

21. Kamps, U. (1995a). A Concept of Generalized Order Statistics. Stuttgart: B.G. Teubner.

22. Kamps, U. (1995b). A concept of generalized order statistics. Journal of Statistical Planning and Inference 48: 1-23.

23. Kamps, U. \& Cramer, E. (2001). On distributions of generalized order statistics. Statistics 35: 269-280.

24. Karlin, S. (1968). Total Positivity. Stanford, CA: Stanford University Press.

25. Marshall, A.W. \& Olkin, I. (2007). Life Distributions. New York: Springer.

26. Nevzorov, V. (2001). Records: Mathematical Theory. Providence, RI: American Mathematical Society.

27. Pellerey, F., Shaked, M. \& Zinn, J. (2000). Nonhomogeneous Poisson processes and logconcavity. Probability in the Engineering and Informational Sciences 14: 353-373.

28. Prékopa, A. (1973). On logarithmic concave measures and functions. Acta Scientiarum Mathematicarum 34: 335-343.

29. Raqab, M.Z. \& Amin, W.A. (1997). A note on reliability properties of $k$-record statistics. Metrika 46 : $245-251$.

30. Shaked, M.\& Shanthikumar, J.G. (2007). Stochastic Orders. New York: Springer.

31. Tavangar, M. \& Asadi, M. (2012). Some unified characterization results on the generalized Pareto distributions based on generalized order statistics. Metrika 75: 997-1007. 